\title{
Congenital Skin Disorder
}

National Cancer Institute

\section{Source}

National Cancer Institute. Congenital Skin Disorder. NCI Thesaurus. Code 697174.

A skin abnormality that is present at birth or detected in the neonatal period. 